\title{
Nonexistence of stable solutions for quasilinear Schrödinger equation
}

\author{
Lijuan Chen ${ }^{1,2^{*}}$ (D), Caisheng Chen ${ }^{1}$, Hongwei Yang ${ }^{3}$ and Hongxue Song ${ }^{1}$
}

${ }^{\text {"Correspondence: }}$

chenlijuan@ycit.cn

${ }^{1}$ College of Science, Hohai University, Nanjing, P.R. China

${ }^{2}$ Yancheng Institute of Technology, Yancheng, P.R. China

Full list of author information is available at the end of the article

\section{Abstract}

In this paper, we study the nonexistence of stable solutions for the quasilinear Schrödinger equation

$$
-\Delta u-\left[\Delta\left(1+u^{2}\right)^{1 / 2}\right] \frac{u}{2\left(1+u^{2}\right)^{1 / 2}}=h(x)|u|^{q-1} u, \quad x \in R^{N},
$$

where $N \geq 3, q \geq 5 / 2$ and the function $h(x)$ is continuous and positive in $R^{N}$. Under suitable assumptions on $h(x)$ and $q$, we prove that Eq. (0.1) has no nonnegative and stable solutions.

Keywords: Nonexistence of solution; Quasilinear Schrödinger equation; Stable solution

\section{Introduction and main results}

In this paper we are interested in the nonexistence of stable solutions to the quasilinear Schrödinger equation

$$
-\Delta u-\left[\Delta\left(1+u^{2}\right)^{1 / 2}\right] \frac{u}{2\left(1+u^{2}\right)^{1 / 2}}=h(x)|u|^{q-1} u, \quad x \in R^{N}
$$

where $N \geq 3$ and $q \geq 5 / 2$. Equation (1.1) can be obtained as a stationary problem of the modified Schrödinger equation

$$
i z_{t}=-\Delta z+W(x) z-h\left(x,|z|^{2}\right) z-\left[\Delta l\left(|z|^{2}\right)\right] l^{\prime}\left(|z|^{2}\right) z, \quad x \in R^{N}
$$

where $z: R \times R^{N} \rightarrow \mathbb{C}, W: R^{N} \rightarrow R$ is a given potential, $h$ and $l$ are real functions.

It is well known that the standing wave solutions of the form $z(t, x)=\exp (-i \omega t) u(x)$ satisfy (1.2) if and only if the real function $u(x)$ solves the equation of elliptic type

$$
-\Delta u+V(x) u-\left[\Delta l\left(u^{2}\right)\right] l^{\prime}\left(u^{2}\right) u=g(x, u), \quad x \in R^{N}
$$

where $V(x)=W(x)-\omega, \omega \in R$ and $g(x, u) \equiv h\left(x, u^{2}\right) u$.

Quasilinear Schrödinger equations as in (1.3) appear naturally in mathematical physics and have been derived as models of several physical phenomena corresponding to various

\section{Springer}

(c) The Author(s) 2018. This article is distributed under the terms of the Creative Commons Attribution 4.0 International License (http://creativecommons.org/licenses/by/4.0/), which permits unrestricted use, distribution, and reproduction in any medium, provided you give appropriate credit to the original author(s) and the source, provide a link to the Creative Commons license, and indicate if changes were made. 
types of nonlinear term $l$. When $l(s)=s$, we get the superfluid film equation in plasma physics [16]:

$$
-\Delta u+V(x) u-\Delta\left(u^{2}\right) u=g(x, u), \quad x \in R^{N} .
$$

In the case $l(s)=(1+s)^{1 / 2},(1.3)$ turns into the following equation:

$$
-\Delta u+V(x) u-\left[\Delta\left(1+u^{2}\right)^{\frac{1}{2}}\right] \frac{u}{2\left(1+u^{2}\right)^{\frac{1}{2}}}=g(x, u), \quad x \in R^{N},
$$

which models the self-channeling of a high-power ultrashort laser in matter, see $[2,10]$.

The existence of positive solutions for (1.5) has been extensively studied recently. In [23], the authors proved that (1.5) has a positive solution under the assumptions: $g(x, u)=$ $\lambda|u|^{p-1} u, \sqrt{6}-1 \leq p<\frac{N+2}{N-2}, \lambda>0$ and $0<\inf _{x \in \mathbb{R}^{N}} V(x) \leq V(x) \leq V(\infty):=\lim _{|x| \rightarrow \infty} V(x)<$ $\infty$.

Li [20] studied the existence of quasilinear Schrödinger equations of the form

$$
-\Delta u+V(x) u-\left[\Delta\left(1+u^{2}\right)^{\alpha / 2}\right] \frac{\alpha u}{2\left(1+u^{2}\right)^{(2-\alpha) / 2}}=g(x, u), \quad x \in R^{N},
$$

where the parameter $\alpha \in[1,2]$ and the functions $V(x), g(x, u)$ are 1-periodic in $x_{j}$ for $j=$ $1,2, \ldots, N$.

Similar works can be found in $[6,7,11,24,27]$ and the references therein. It is noted that, in the above works, one always assumes that the potential function $V(x) \geq 0$ and $V(x) \not \equiv 0$ in $\mathbb{R}^{N}$.

On the other hand, the nonexistence of solutions and either the stable or unstable solutions for Lane-Emden problems are investigated to some extent. The results can be found in $[1,3,8,14,15,18,19,21,25,26,28]$, and the references therein. To the best of our knowledge, there are no results on the nonexistence of solutions for (1.1). Motivated by [4, 5, $9,13,17,22]$, our purpose in this paper is to study the nonexistence of nonnegative and stable solutions of (1.1) under some assumptions on the weighted function $h(x)$ and the exponent $q$.

Usually, we make the change of variables $z=f^{-1}(u)$, where $f$ is defined by

$$
f^{\prime}(t)=\left[1+\frac{f^{2}(t)}{2\left(1+f^{2}(t)\right)}\right]^{-1 / 2}=\sqrt{2}\left(1+f^{2}(t)\right)^{\frac{1}{2}}\left(2+3 f^{2}(t)\right)^{-\frac{1}{2}}, \quad t \geq 0, f(0)=0
$$

and by $f(t)=-f(-t)$ on $(-\infty, 0]$.

Lemma $1.1([20,23])$ The function $f(t)$ satisfies the following properties:

$\left(f_{1}\right) f$ is uniquely defined, odd, increasing, invertible and $C^{\infty}$ in $R=(-\infty,+\infty)$,

$\left(f_{2}\right) 0<f^{\prime}(t) \leq 1, \forall t \in R$,

$\left(f_{3}\right)|f(t)| \leq|t|, \forall t \in R$,

(f $\left.f_{4}\right) \frac{f(t)}{t} \rightarrow 1$ as $t \rightarrow 0$,

$\left(f_{5}\right) f(t) \leq 2 t f^{\prime}(t) \leq 2 f(t), \forall t \in R^{+}=[0, \infty)$,

$\left(f_{6}\right) \lim _{t \rightarrow+\infty} \frac{f(t)}{t}=\sqrt{\frac{2}{3}}$. 
If we take $u=f(z)$ or $z=f^{-1}(u)$, then (1.1) becomes the following semilinear elliptic equation:

$$
-\Delta z=h(x) f^{\prime}(z)|f(z)|^{q-1} f(z), \quad x \in R^{N} .
$$

As usual, we study the existence and the nonexistence of weak solutions of (1.1) via (1.8).

Definition 1.1 ([12]) The function $z \in C_{\mathrm{loc}}^{1, \delta}\left(\mathbb{R}^{N}\right)(0<\delta<1)$ is said to be a weak solution of (1.8) if

$$
\int_{\mathbb{R}^{N}} \nabla z \nabla \zeta d x=\int_{\mathbb{R}^{N}} h(x) g(z) \zeta d x, \quad \forall \zeta \in C_{0}^{1}\left(\mathbb{R}^{N}\right),
$$

where (and in the sequel) $g(z)=f^{\prime}(z)|f(z)|^{q-1} f(z)$. A weak solution $z$ of (1.8) is stable if

$$
\int_{\mathbb{R}^{N}}|\nabla \zeta|^{2} d x \geq \int_{\mathbb{R}^{N}} h(x) g^{\prime}(z) \zeta^{2} d x, \quad \forall \zeta \in C_{0}^{1}\left(\mathbb{R}^{N}\right) .
$$

In other words, the stability condition translates into the fact that the second variation of the energy functional is nonnegative. Thus, all the minima of the functional are stable solutions of (1.8).

As in $[11,20,23]$, we give:

Definition 1.2 A function $u \in \mathcal{D}^{1,2}\left(\mathbb{R}^{N}\right)$ is called a weak solution of (1.1) if $z=f^{-1}(u)$ is a weak solution of (1.8). A weak solution $u$ of (1.1) is stable in $\mathbb{R}^{N}$ if $z=f^{-1}(u)$ is a stable solution of (1.8).

So in order to prove the nonexistence of stable solutions for (1.1), it is sufficient to prove that there is no nonnegative and stable weak solution to (1.8).

The main result in this paper is as follows.

Theorem 1.2 Suppose that the positive function $h(x) \in C_{\mathrm{loc}}^{1}\left(\mathbb{R}^{N}\right)$ is such that there exist $a>-2, a_{0}>0$ and $R_{0}>0$ such that

$$
h(x) \geq a_{0}|x|^{a}, \quad \forall|x| \geq R_{0} .
$$

Denote

$$
\gamma_{0}(a):=6+2 a, \quad \gamma_{\infty}(a):=10+4 a
$$

Let $q_{c}$ be the positive root of the equation $N=X(q)$ with

$$
X(t)=\frac{2\left[t+k_{0}(t)\right]+a\left[k_{0}(t)+1\right]}{t-1}, \quad t \geq 5 / 2,
$$

and

$$
k_{0}(t)=2 t-3+\sqrt{4 t^{2}-12 t+5}, \quad t \geq 5 / 2 .
$$


Moreover, let $u \in C_{\text {loc }}^{1, \delta}\left(\mathbb{R}^{N}\right)(0<\delta<1)$ be a nonnegative and stable solution of $(1.1)$. Then, we have $u \equiv 0$ in $\mathbb{R}^{N}$ if one of the following conditions is satisfied:

$\left(A_{1}\right) q \geq \frac{5}{2}$ and $3 \leq N \leq \gamma_{0}(a)$;

$\left(A_{2}\right) q>q_{c}$ and $\gamma_{0}(a)<N<\gamma_{\infty}(a)$.

Remark 1.3 When $N \in\left(\gamma_{0}, \gamma_{\infty}\right)$, it is not difficult to get

$$
q_{c}=\frac{6-d^{2}+\sqrt{16-3 d^{2}}}{4-d^{2}} \quad \text { with } d=\frac{N-6-2 a}{2+a} .
$$

The condition $\gamma_{0}<N<\gamma_{1}$ implies that $d \in(0,2)$ and $q_{c}>5 / 2$.

\section{Proof of Theorem 1.2}

In order to prove the nonexistence of solution to (1.8), we use the test function method, which has been used in $[5,9]$ to deal with the $m$-Laplace equation. The proof is by contradiction which involves obtaining an a priori estimate for a solution of (1.8) by carefully choosing a special test function and then applying the scaling argument. We first establish:

Lemma 2.1 Assume $q>1$. Let $f(t)$ be the function defined by (1.7) and $k>1$. Suppose $g(t)=f^{\prime}(t)|f(t)|^{q-1} f(t)$ and

$$
G(t)=\left(\frac{g(t)}{g^{\prime}(t)} t^{k}\right)^{1 / 2}, \quad \forall t \geq 0 .
$$

Then there exist $d_{1}, d_{1}^{\prime}>0$ such that

$$
d_{1}^{\prime} t^{k+1} \leq G^{2}(t) \leq d_{1} t^{k+1}, \quad \forall t \geq 0
$$

Furthermore, if $q \geq 2$, we have for all $t \geq 0$,

$$
\left(G^{\prime}(t)\right)^{2} \leq \alpha_{k} t^{k-1}, \quad \text { where } \alpha_{k}=\frac{(k+1)^{2}}{2 q-1} .
$$

Proof Let $f=f(t)(t \geq 0)$. Direct computation shows that

$$
g^{\prime}(t)=2 \beta(t) f^{q-1}\left[2+3 f^{2}\right]^{-2}, \quad \text { where } \beta(t)=2 q+(5 q-1) f^{2}(t)+3 q f^{4}(t)
$$

and

$$
G^{2}(t)=\frac{g(t)}{g^{\prime}(t)} t^{k}=\frac{f t^{k}\left(1+f^{2}\right)^{\frac{1}{2}}\left(2+3 f^{2}\right)^{\frac{3}{2}}}{\sqrt{2} \beta(t)} .
$$

From $f(0)=0$, as well as $\left(f_{4}\right)$ and $\left(f_{6}\right)$ in Lemma 1.1, it follows that

$$
\lim _{t \rightarrow 0^{+}} \frac{G^{2}(t)}{t^{k+1}}=\frac{1}{q} \text { and } \lim _{t \rightarrow+\infty} \frac{G^{2}(t)}{t^{k+1}}=\frac{1}{q} .
$$

Furthermore, noticing the fact that the function $H(t)=t^{-k-1} G^{2}(t)$ is positive and continuous on any bounded interval $[c, d] \subset(0,+\infty)$, we obtain (2.2) from (2.6). 
In the following we prove (2.3). First, we have from (2.5) that

$$
\sqrt{2} \beta(t) G^{2}(t)=f t^{k}\left(1+f^{2}\right)^{\frac{1}{2}}\left(2+3 f^{2}\right)^{\frac{3}{2}}, \quad t \geq 0 .
$$

Differentiating (2.7) with respect to $t$ gives

$$
\begin{gathered}
2 \sqrt{2} G(t) G^{\prime}(t) \beta(t)+\sqrt{2} G^{2}(t) \beta^{\prime}(t)=\left[f t^{k}\left(1+f^{2}\right)^{\frac{1}{2}}\left(2+3 f^{2}\right)^{\frac{3}{2}}\right]^{\prime} \\
=k t^{k-1} f\left(1+f^{2}\right)^{\frac{1}{2}}\left(2+3 f^{2}\right)^{\frac{3}{2}}+\sqrt{2} t^{k}\left(2+16 f^{2}+15 f^{4}\right) .
\end{gathered}
$$

Since

$$
\sqrt{2} G^{2}(t) \beta^{\prime}(t)=2 \sqrt{2} t^{k} f^{2}\left(1+f^{2}\right)\left(2+3 f^{2}\right)\left(5 q-1+6 q f^{2}\right) \beta^{-1}(t)
$$

we have from (2.5), (2.8) and (2.9) that

$$
8\left(G^{\prime}(t)\right)^{2}=t^{k-1}\left(A_{1}(t)+A_{2}(t)+A_{3}(t)\right), \quad \forall t \geq 0,
$$

where

$$
\begin{aligned}
& A_{1}(t)=\frac{\sqrt{2} k^{2} f\left(1+f^{2}\right)^{\frac{1}{2}}\left(2+3 f^{2}\right)^{\frac{3}{2}}}{t \beta(t)}, \\
& A_{2}(t)=\frac{4 k \gamma(t)}{\beta^{2}(t)}, \\
& A_{3}(t)=\frac{2 \sqrt{2} t \gamma^{2}(t)}{f \beta^{3}\left(1+f^{2}\right)^{\frac{1}{2}}\left(2+3 f^{2}\right)^{\frac{3}{2}}}
\end{aligned}
$$

and

$$
\begin{aligned}
\gamma(t) & =\beta(t)\left(2+16 f^{2}+15 f^{4}\right)-2 f^{2}\left(1+f^{2}\right)\left(2+3 f^{2}\right)\left(5 q-1+6 q f^{2}\right) \\
& =4 q+2(11 q+1) f^{2}+6(7 q-1) f^{4}+3(11 q-3) f^{6}+9 q f^{8} .
\end{aligned}
$$

Using the property $\left(f_{5}\right)$ in Lemma 1.1 , we have

$$
\frac{f(t)}{t} \leq 2 f^{\prime}(t)=2 \sqrt{2}\left(1+f^{2}\right)^{\frac{1}{2}}\left(2+3 f^{2}\right)^{-\frac{1}{2}}, \quad \forall t \geq 0,
$$

and then

$$
A_{1}(t) \leq \frac{4 k^{2}\left(1+f^{2}\right)\left(2+3 f^{2}\right)}{\beta(t)}=\frac{4 k^{2}\left(2+5 f^{2}+3 f^{4}\right)}{2 q+(5 q-1) f^{2}+3 q f^{4}} \leq \frac{20 k^{2}}{5 q-1}, \quad \forall t \geq 0 .
$$

On the other hand, directive computation gives

$$
\beta^{2}(t)=4 q^{2}+4 q(5 q-1) f^{2}+\left(37 q^{2}-10 q+1\right) f^{4}+6 q(5 q-1) f^{6}+9 q^{2} f^{8}
$$

Then an application of (2.12) and (2.15) yields $\gamma(t) \leq \frac{3}{2 q} \beta^{2}(t)$, and so

$$
A_{2}(t) \leq \frac{6 k}{q}, \quad \forall t \geq 0
$$


Furthermore, we see from $\left(f_{5}\right)$ in Lemma 1.1 that

$$
\frac{t}{f(t)} \leq \frac{1}{f^{\prime}(t)}=2^{-\frac{1}{2}}\left(1+f^{2}\right)^{-\frac{1}{2}}\left(2+3 f^{2}\right)^{\frac{1}{2}}
$$

and then

$$
A_{3}(t) \leq \frac{2 \gamma^{2}(t)}{\beta^{3}(t)\left(2+5 f^{2}+3 f^{4}\right)}, \quad \forall t \geq 0
$$

We now claim

$$
\frac{\gamma^{2}(t)}{\beta^{3}(t)\left(2+5 f^{2}+3 f^{4}\right)} \leq \frac{2}{q}, \quad \forall t \geq 0
$$

In fact, we have

$$
\begin{aligned}
\gamma^{2}(t)= & 16 q^{2}+16 q(11 q+1) f^{2}+4\left(205 q^{2}+10 q+1\right) f^{4}+24\left(88 q^{2}-7 q-1\right) f^{6} \\
& +24 q(137 q-32) f^{8}+36\left(88 q^{2}-31 q+3\right) f^{10}+9\left(205 q^{2}-78 q+9\right) f^{12} \\
& +54 q(11 q-3) f^{14}+81 q^{2} f^{16}
\end{aligned}
$$

and

$$
\begin{aligned}
\left(2+5 f^{2}+3 f^{4}\right) \beta^{3}(t)= & \left(2+5 f^{2}+3 f^{4}\right)\left[2 q+(5 q-1) f^{2}+3 q f^{4}\right]^{3} \\
= & 16 q^{3}+8 q^{2}(20 q-3) f^{2}+12 q\left(58 q^{2}-15 q+1\right) f^{4} \\
& +2\left(860 q^{3}-279 q^{2}+30 q-1\right) f^{6} \\
& +\left(2641 q^{3}-915 q^{2}+111 q-5\right) f^{8} \\
& +3\left(860 q^{3}-279 q^{2}+30 q-1\right) f^{10} \\
& +27 q\left(58 q^{2}-15 q+1\right) f^{12}+27 q^{2}(20 q-3) f^{14} \\
& +81 q^{3} f^{16}
\end{aligned}
$$

Clearly, it is not difficult to verify (2.19) by the use of (2.20) and (2.21). Then, from (2.10), (2.14), (2.16), (2.18) and (2.19), we obtain

$$
\left(G^{\prime}(t)\right)^{2} \leq t^{k-1}\left(\frac{5 k^{2}}{2(5 q-1)}+\frac{3 k}{4 q}+\frac{1}{2 q}\right) \leq \frac{(k+1)^{2}}{2 q-1} t^{k-1}, \quad \forall t \geq 0,
$$

and the proof of Lemma 2.1 is completed.

On the other hand, for the function $g(t)$ defined in Lemma 2.1, we have the following result.

Lemma 2.2 Assume $q>1$. Let $f(t)$ be the function defined by (1.7) and $k>1$. Suppose $g(t)=f^{\prime}(t)|f(t)|^{q-1} f(t)$. Then, there exists $M_{1}>0$ such that

$$
\left|\frac{t^{q}}{g(t)}\right| \leq M_{1}, \quad \forall t>0
$$


Proof Obviously, the function $Y(t)=\frac{t^{q}}{g(t)}$ is continuous on $(0,+\infty)$. From $\left(f_{4}\right)$ and $\left(f_{6}\right)$ in Lemma 1.1, we derive

$$
\lim _{t \rightarrow 0^{+}} Y(t)=\lim _{t \rightarrow 0^{+}} \frac{t^{q}\left(2+3 f^{2}\right)^{\frac{1}{2}}}{\sqrt{2}\left(1+f^{2}\right)^{\frac{1}{2}} f^{q}(t)}=\lim _{t \rightarrow 0^{+}} \frac{t^{q}}{f^{q}(t)}=1
$$

and

$$
\lim _{t \rightarrow+\infty} Y(t)=\left(\frac{3}{2}\right)^{\frac{1}{2}} \lim _{t \rightarrow+\infty} \frac{t^{q}}{f^{q}(t)}=\left(\frac{3}{2}\right)^{(q+1) / 2} .
$$

Then the conclusion (2.23) follows, and the proof of Lemma 2.2 is completed.

Lemma 2.3 Let $z \in C_{\text {loc }}^{1, \delta}\left(\mathbb{R}^{N}\right)(0<\delta<1)$ be a nonnegative and stable weak solution of (1.8) with $q \geq 5 / 2$. Then for every $k \in\left(1, k_{0}(q)\right)$, where $k_{0}(t)$ is defined by (1.14), there exists a constant $C=C(q, k)$ such that

$$
\int_{\mathbb{R}^{N}} h(x) g(z) z^{k} \varphi^{2} d x \leq C \int_{\mathbb{R}^{N}}|h(x)|^{-\frac{k+1}{q-1}}|\nabla \varphi|^{\frac{2(q+k)}{q-1}} \varphi^{-\frac{2(k+1)}{q-1}} d x,
$$

where $\varphi=\varphi(x) \in C_{0}^{1}\left(\mathbb{R}^{N}\right)$ is a nonnegative cut-off function, in which $\varphi(x)=\varphi_{0}\left(\frac{|x|}{R}\right)$ with $R>0, \varphi_{0}(s) \in C_{0}^{1}\left(\mathbb{R}^{+}\right), 0 \leq \varphi_{0}(s) \leq 1$, and it is defined by

$$
\varphi_{0}(s)= \begin{cases}1, & 0 \leq s \leq 1 \\ 2(2-s)^{m}-(2-s)^{2 m}, & 1<s<2 \\ 0, & s>2\end{cases}
$$

with $m=\frac{q+k}{q-1}>1$.

Remark 2.4 It is not difficult to verify that $0 \leq \varphi_{0}(s) \leq 1$ and $\left|\varphi_{0}^{\prime}(s)\right| \leq \beta_{0} \varphi_{0}^{1-1 / m}(s)$ with $\beta_{0}=2^{1 / m} m$.

Proof Let $z \in C_{\text {loc }}^{1, \delta}\left(\mathbb{R}^{N}\right)$ be a nonnegative and stable weak solution of (1.8) and $k>1$. Setting $\zeta=z^{k} \varphi^{2}$ in (1.9), we find

$$
k \int_{\mathbb{R}^{N}}|\nabla z|^{2} z^{k-1} \varphi^{2} d x \leq 2 \int_{\mathbb{R}^{N}}|\nabla z||\nabla \varphi| z^{k} \varphi d x+\int_{\mathbb{R}^{N}} h(x) g(z) z^{k} \varphi^{2} d x
$$

Applying Young's inequality with parameter $\epsilon \in(0,1)$, we obtain

$$
2 \int_{\mathbb{R}^{N}}|\nabla z||\nabla \varphi| z^{k} \varphi d x \leq \epsilon \int_{\mathbb{R}^{N}}|\nabla z|^{2} z^{k-1} \varphi^{2} d x+C \int_{\mathbb{R}^{N}}|\nabla \varphi|^{2} z^{k+1} d x
$$

Here and in the sequel, let $C$ be a positive constant depending on $\epsilon$ and $q, k$, which may vary from line to line. Then it follows from (2.28) and (2.29) that

$$
(k-\epsilon) \int_{\mathbb{R}^{N}}|\nabla z|^{2} z^{k-1} \varphi^{2} d x \leq \int_{\mathbb{R}^{N}} h(x)|g(z)| z^{k} \varphi^{2} d x+C \int_{\mathbb{R}^{N}}|\nabla \varphi|^{2} z^{k+1} d x .
$$


On the other hand, taking $\zeta=G(z) \varphi$ in (1.10), we find

$$
|\nabla \zeta|^{2}=\varphi^{2}\left(G^{\prime}(z)\right)^{2}|\nabla z|^{2}+2 \varphi G^{\prime}(z) G(z) \nabla z \nabla \varphi+G^{2}(z)|\nabla \varphi|^{2}
$$

and then

$$
\begin{aligned}
\int_{\mathbb{R}^{N}} h(x) g(z) z^{k} \varphi^{2} d x \leq & \int_{\mathbb{R}^{N}}|\nabla z|^{2}\left|G^{\prime}(z)\right|^{2} \varphi^{2} d x+2 \int_{\mathbb{R}^{N}}|\nabla z||\nabla \varphi|\left|G^{\prime}(z) G(z)\right| \varphi d x \\
& +\int_{\mathbb{R}^{N}}|\nabla \varphi|^{2} G^{2}(z) d x .
\end{aligned}
$$

By Young's inequality with $\epsilon>0$, one derives

$$
\begin{aligned}
2 \int_{\mathbb{R}^{N}}|\nabla z||\nabla \varphi|\left|G^{\prime}(z) G(z)\right| \varphi d x \leq & \epsilon \int_{\mathbb{R}^{N}}|\nabla z|^{2}\left|G^{\prime}(z)\right|^{2} \varphi^{2} d x \\
& +C \int_{\mathbb{R}^{N}}|\nabla \varphi|^{2}|G(z)|^{2} d x .
\end{aligned}
$$

Then, it follows from (2.2), (2.3), (2.30) and (2.32) that

$$
\begin{aligned}
\int_{\mathbb{R}^{N}} h(x) g(z) z^{k} \varphi^{2} d x \leq & (1+\epsilon) \int_{\mathbb{R}^{N}}\left|G^{\prime}(z)\right|^{2}|\nabla z|^{2} \varphi^{2} d x+C \int_{\mathbb{R}^{N}}|\nabla \varphi|^{2}|G(z)|^{2} d x \\
\leq & \alpha_{k}(1+\epsilon) \int_{\mathbb{R}^{N}}|\nabla z|^{2} \varphi^{2} z^{k-1} d x+C \int_{\mathbb{R}^{N}}|\nabla \varphi|^{2}|G(z)|^{2} d x \\
\leq & \frac{\alpha_{k}(1+\epsilon)}{k-\epsilon} \int_{\mathbb{R}^{N}} h(x) g(z) z^{k} \varphi^{2} d x+C \int_{\mathbb{R}^{N}}|\nabla \varphi|^{2} z^{k+1} d x \\
& +C \int_{\mathbb{R}^{N}}|G(z)|^{2}|\nabla \varphi|^{2} d x \\
\leq & \frac{\alpha_{k}(1+\epsilon)}{k-\epsilon} \int_{\mathbb{R}^{N}} h(x) g(z) z^{k} \varphi^{2} d x+C_{1} \int_{\mathbb{R}^{N}}|\nabla \varphi|^{2} z^{k+1} d x
\end{aligned}
$$

Then one sees from (2.34) that

$$
\delta_{\epsilon} \int_{\mathbb{R}^{N}} h(x) g(z) z^{k} \varphi^{2} d x \leq C \int_{\mathbb{R}^{N}}|\nabla \varphi|^{2} z^{k+1} d x
$$

where

$$
\delta_{\epsilon}=1-\frac{\alpha_{k}(1+\epsilon)}{k-\epsilon}=1-\frac{(1+\epsilon)(k+1)^{2}}{(k-\epsilon)(2 q-1)} .
$$

Clearly,

$$
\delta_{0}=\lim _{\epsilon \rightarrow 0+} \delta_{\epsilon}=1-\frac{(k+1)^{2}}{k(2 q-1)} .
$$

Moreover, the elementary inequality $\delta_{0}>0$ implies that $k \in\left(1, \frac{k_{0}(q)}{2}\right)$, where $k_{0}(t)$ is defined by (1.14). Now, an application of (2.35) yields

$$
\int_{\mathbb{R}^{N}} h(x) g(z) z^{k} \varphi^{2} d x \leq C \int_{\mathbb{R}^{N}}|\nabla \varphi|^{2} z^{k+1} d x
$$


Let $\lambda=\frac{q+k}{k+1}, \lambda^{\prime}=\frac{q+k}{q-1}$. Then it follows from Lemma 2.2 that, if $z=z(x)$ is nonnegative in $\mathbb{R}^{N}$, the function $\psi(x)=z^{(k+1) \lambda^{\prime}}\left(g(z) z^{k}\right)^{-\frac{\lambda^{\prime}}{\lambda}}=\left(\frac{z^{q}}{g(z)}\right)^{\frac{k+1}{q-1}}$ is nonnegative and bounded in $\mathbb{R}^{N}$. Moreover, we obtain from the Hölder inequality, (2.23) and (2.38) that

$$
\begin{aligned}
& \int_{\mathbb{R}^{N}} h(x) g(z) z^{k} \varphi^{2} d x \\
& \leq C \int_{\mathbb{R}^{N}}|\nabla \varphi|^{2} z^{k+1} d x \\
& \leq C\left(\int_{\mathbb{R}^{N}} h(x) g(z) z^{k} \varphi^{2} d x\right)^{\frac{1}{\lambda}}\left(\int_{\mathbb{R}^{N}}|h(x)|^{-\frac{\lambda^{\prime}}{\lambda}}|\nabla \varphi|^{2 \lambda^{\prime}} \varphi^{-\frac{2 \lambda^{\prime}}{\lambda}} z^{(k+1) \lambda^{\prime}}\left(g z^{k}\right)^{-\frac{\lambda^{\prime}}{\lambda}} d x\right)^{\frac{1}{\lambda^{\prime}}} \\
& \quad \leq C\left(\int_{\mathbb{R}^{N}} h(x) g(z) z^{k} \varphi^{2} d x\right)^{\frac{1}{\lambda}}\left(\int_{\mathbb{R}^{N}}|h(x)|^{-\frac{\lambda^{\prime}}{\lambda}}|\nabla \varphi|^{2 \lambda^{\prime}} \varphi^{-\frac{2 \lambda^{\prime}}{\lambda}} d x\right)^{\frac{1}{\lambda^{\prime}}} \cdot
\end{aligned}
$$

Obviously, inequality (2.39) implies

$$
\int_{\mathbb{R}^{N}} h(x) g(z) z^{k} \varphi^{2} d x \leq C \int_{\mathbb{R}^{N}}|h(x)|^{-\frac{\lambda^{\prime}}{\lambda}}|\nabla \varphi|^{2 \lambda^{\prime}} \varphi^{-\frac{2 \lambda^{\prime}}{\lambda}} d x
$$

and (2.26) follows.

Proof of Theorem 1.2 Setting $x=R \xi$ in (2.26), we get

$$
\begin{aligned}
\int_{\mathbb{R}^{N}} h(x) g(z) z^{k} \varphi^{2} d x & \leq C \int_{\mathbb{R}^{N}}|h(x)|^{-\frac{k+1}{q-1}}|\nabla \varphi|^{\frac{2(q+k)}{q-1}} \varphi^{-\frac{2(k+1)}{q-1}} d x \\
& \leq C R^{\theta} \int_{1 \leq|\xi| \leq 2}\left[\frac{\left|\varphi_{0}^{\prime}(|\xi|)\right|}{1-\frac{q-1}{q+k}(|\xi|)}\right]^{\frac{2(q+k)}{q-1}} d \xi \leq C R^{\theta} \beta_{0}^{\frac{2(q+k)}{q-1}}
\end{aligned}
$$

where assumption (1.11) on $h(x)$ has been used, $C$ is a positive constant independent of $R$, and

$$
\theta=N-\frac{2(q+k)+a(k+1)}{q-1}
$$

Clearly, if $\theta<0$, the desired result follows by letting $R \rightarrow \infty$ in (2.41). In the following, we will show that some appropriate $k=k(q)$ can be chosen such that $\theta<0$. Let $X(t)$ be the function defined in (1.13). Obviously, we have

$$
\lim _{t \rightarrow 5 / 2} X(t)=\gamma_{0}(a):=6+2 a, \quad \lim _{t \rightarrow+\infty} X(t)=\gamma_{\infty}(a):=10+4 a
$$

Note that $X(t)=2+(2+a) Y(t)(t \geq 5 / 2)$ with

$$
Y(t)=\frac{k_{0}(t)+1}{t-1}=2+\frac{\sqrt{4 t^{2}-12 t+5}}{t-1}
$$

and

$$
X^{\prime}(t)=\frac{(2+a)(2 t+1)}{(t-1)^{2}}\left(4 t^{2}-12 t+5\right)^{-1 / 2}>0, \quad \forall t>5 / 2 .
$$


So, the function $X(t)$ is increasing and $6+2 a<X(t)<10+4 a$ for $t>5 / 2$.

Therefore, if $N \leq 6+2 a$, we have $N<X(t)$ for any $t>5 / 2$. Hence, if we fix $k \in\left(1, k_{0}(t)\right)$ suitably near $k_{0}(t)$, we obtain

$$
N<\frac{2\left[t+k_{0}(t)\right]+a\left[k_{0}(t)+1\right]}{t-1} .
$$

For this reason, the desired result follows by letting $R \rightarrow \infty$ in (2.41).

Assume now $\gamma_{0}(a)<N<\gamma_{\infty}(a)$. Since $X(t)$ is increasing, we get in this case a critical value $q_{c}>5 / 2$ such that $N<X(q)$ for $q_{c}<q$. From this, the desired result follows again by letting $R \rightarrow \infty$ in (2.41). Clearly, $q_{c}$ may be determined from the equation $N=X\left(q_{c}\right)$. Then we complete the proof of Theorem 1.2.

\title{
3 Concluding remarks
}

In this paper, we have considered a model described by the quasilinear Schrödinger equation. Nonexistence of stable solutions is proved.

\author{
Acknowledgements \\ The authors thank the reviewers for careful reading of the paper. \\ Funding \\ Support from the Fundamental Research Funds for the Central Universities of China (2015B31014) and by National \\ Natural Science Foundation of China (No. 11571092) as well as by the China Postdoctoral Science Foundations (Grant No. \\ 2017M611664) and the Nature Science Foundation of the Jiangsu Higher Education Institutions of China (No. \\ 18KJB110030) is acknowledged.
}

\section{Availability of data and materials}

Data sharing not applicable to this article as no datasets were generated or analyzed during the current study.

\section{Competing interests}

The authors declare that there are no competing interests among them.

\section{Authors' contributions}

All authors contributed equally to the writing of this paper. All authors read and approved the final manuscript.

\section{Author details}

${ }^{1}$ College of Science, Hohai University, Nanjing, P.R. China. ${ }^{2}$ Yancheng Institute of Technology, Yancheng, P.R. China. ${ }^{3}$ Shan Dong University of Science and Technology, Qingdao, P.R. China.

\section{Publisher's Note}

Springer Nature remains neutral with regard to jurisdictional claims in published maps and institutional affiliations.

Received: 2 June 2018 Accepted: 23 October 2018 Published online: 06 November 2018

\section{References}

1. Birindelli, I., Galise, G., Leoni, F.: Liouville theorems for a family of very degenerate elliptic nonlinear operators. Nonlinear Anal. 161, 198-211 (2017)

2. Brandi, H., Manus, C., Mainfray, G., Lehner, T., Bonnaud, G.: Relativistic and ponderomotive self-focusing of a laser beam in a radially inhomogeneous plasma. Phys. Fluids B 5, 3539-3550 (1993)

3. Brezis, H., Vázquez, J.-L.: Blow-up solutions of some nonlinear elliptic problems. Rev. Mat. Univ. Complut. Madr. 10(2), $443-469$ (1997)

4. Chen, C.S.: Liouville type theorem for stable solutions of $p$-Laplace equation in $R^{N}$. Appl. Math. Lett. 68, 62-67 (2017)

5. Chen, C.S., Song, H.X., Yang, H.W.: Liouville type theorems for stable solutions of $p$-Laplace equation in $R^{N}$. Nonlinear Anal. 160, 44-52 (2017)

6. Chen, C.S., Yang, H.W.: Multiple Solutions for a Class of Quasilinear Schrödinger systems in $R^{N}$. Bull. Malays. Math. Sci. Soc. https://doi.org/10.1007/s40840-017-0502-z

7. Chen, J.H., Tang, X.H., Cheng, B.T.: Non-Nehari manifold method for a class of generalized quasilinear Schrödinger equations. Appl. Math. Lett. 74, 20-26 (2017)

8. Cowan, C., Fazly, M.: On stable entire solutions of semi-linear elliptic equations with weights. Proc. Am. Math. Soc. $140,2003-2012(2012)$

9. Damascelli, L., Farina, A., Sciunzi, B., Valdinoci, E.: Liouville results for $m$-Laplace equations of Lane-Emden-Fowler type. Ann. Inst. Henri Poincaré, Anal. Non Linéaire 26, 1099-1119 (2009) 
10. De Bouard, A., Hayashi, N., Saut, J.: Global existence of small solutions to a relativistic nonlinear Schrödinger equation. Commun. Math. Phys. 189, 73-105 (1997)

11. Deng, Y.B., Huang, W.T.: Positive ground state solutions for a quasilinear elliptic equation with critical exponent. Discrete Contin. Dyn. Syst. 37(8), 4213-4230 (2017)

12. Dupaigne, L:: Stable Solutions of Elliptic Partial Differential Equations. CRC Press, Boca Raton (2011)

13. Farina, A.: On the classification of solutions of Lane-Emden equation on unbounded domains of $R^{N}$. J. Math. Pures Appl. 87, 537-561 (2007)

14. Ghergu, M., Radulescu, V:: Multi-parameter bifurcation and asymptotics for the singular Lane-Emden-Fowler equation with a convection term. Proc. R. Soc. Edinb., Sect. A 135(1), 61-83 (2005)

15. Ghergu, M., Radulescu, V:: Singular Elliptic Problems: Bifurcation and Asymptotic Analysis. Oxford Lecture Series in Mathematics and Its Applications, vol. 37. Oxford University Press, Oxford (2008)

16. Kurihura, S.: Large-amplitude quasi-solitons in superfluids films. J. Phys. Soc. Jpn. 50, 3262-3267 (1981)

17. Le, P.: Liouville theorems for stable solutions of $p$-Laplace equations with convex nonlinearities. J. Math. Anal. Appl. 443, 431-444 (2016)

18. Le, P., Ho, V:: On stable solutions to weighted quasilinear problems of Lane-Emden type. Electron. J. Differ. Equ. 2018, $71(2018)$

19. Le, P., Huynh, N.V., Ho, V.: Classification results for Kirchhoff equations in $R^{N}$. Complex Var. Elliptic Equ. (2018). https://doi.org/10.1080/17476933.2018.1505874

20. Li, G.B.: Positive solution for quasilinear Schrödinger equations with a parameter. Commun. Pure Appl. Anal. 14(5), 1803-1816 (2015)

21. Mironescu, P., Radulescu, V.: The study of a bifurcation problem associated to an asymptotically linear function. Nonlinear Anal. 26(4), 857-875 (1996)

22. Phan, Q., Souplet, P.: Liouville-type theorems and bounds of solutions of Hardy-Hénon equations. J. Differ. Equ. 252, 2544-2562 (2012)

23. Shen, Y.T., Wang, Y: Soliton solutions for generalized quasilinear Schrödinger equations. Nonlinear Anal. 80, 194-201 (2013)

24. Song, H.X., Chen, C.S.: Existence of weak solutions for generalized quasilinear Schrödinger equations. J. Dyn. Control Syst. 22, 369-383 (2016)

25. Trabelsi, M., Trabelsi, N.: Singular limit solutions for a 2-dimensional semilinear elliptic system of Liouville type. Adv. Nonlinear Anal. 5(4), 315-329 (2016)

26. Wei, Y.F., Chen, C.S., Yang, H.W., Song, H.X.: Multiplicity of solutions for a class of fractional p-Kirchhoff system with sign-changing weight functions. Bound. Value Probl. 2018, 78 (2018)

27. Yang, J., Wang, Y., Abdelgadir, A.: Soliton solutions for quasilinear Schrödinger equations. J. Math. Phys. 54, 071502 (2013)

28. Yuan, Y., Chen, C.S., Yang, H.W.: Existence of positive solutions for a Schrödinger-Poisson system with bounded potential and weighted functions in $R^{3}$. Bound. Value Probl. 2017, 151 (2017)

\section{Submit your manuscript to a SpringerOpen ${ }^{\circ}$ journal and benefit from:}

- Convenient online submission

- Rigorous peer review

- Open access: articles freely available online

- High visibility within the field

- Retaining the copyright to your article

Submit your next manuscript at $\gg$ springeropen.com 